\title{
Self-Assembly of a Structurally Defined Chiro-Optical Peptide- Oligothiophene Hybrid Material
}

\author{
Zeinab Rouhbakhsh, ${ }^{\dagger \dagger}$ Daniel Aili, ${ }^{\dagger}$ Erik Martinsson, ${ }^{\dagger}$ Anna Svärd, ${ }^{\dagger}$ Marcus Bäck, ${ }^{\S}$ \\ Mohammad R. Housaindokht, ${ }^{\ddagger}$ K. K. Peter R. Nilsson, ${ }^{\$(0)}$ and Robert Selegård*, ${ }^{* \dagger}$ \\ ${ }^{\dagger}$ Laboratory of Molecular Materials, Division of Molecular Physics, Department of Physics, Chemistry and Biology, and ${ }^{\S}$ Division of \\ Chemistry, Department of Physics, Chemistry and Biology, Linköping University, 58183 Linköping, Sweden \\ ${ }^{\ddagger}$ Biophysical Chemistry Laboratory, Department of Chemistry, Faculty of Science, Ferdowsi University of Mashhad, $91775-1436$ \\ Mashhad, Iran
}

Supporting Information

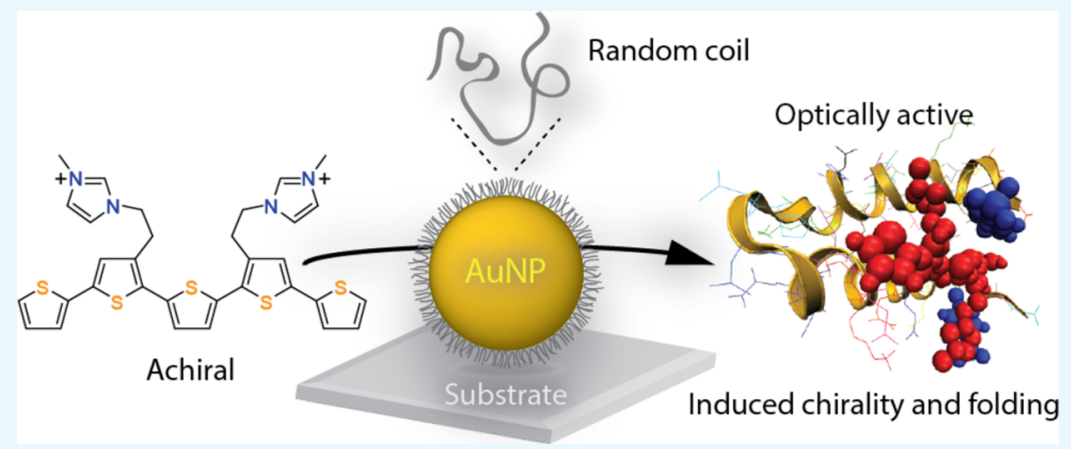

ABSTRACT: Conducting polymers are routinely used in optoelectronic biomaterials, but large polymer polydispersity and poor aqueous compatibility complicate integration with biomolecular templates and development of discrete and defined supramolecular complexes. Herein, we report on a chiro-optical hybrid material generated by the self-assembly of an anionic peptide and a chemically defined cationic pentameric thiophene in aqueous environment. The peptide acts as a stereochemical template for the thiophene and adopts an $\alpha$-helical conformation upon association, inducing optical activity in the thiophene $\pi-\pi^{*}$ transition region. Theoretical calculations confirm the experimentally observed induced structural changes and indicate the importance of electrostatic interactions in the complex. The association process is also probed at the substrate-solvent interface using peptide-functionalized gold nanoparticles, indicating that the peptide can also act as a scaffold when immobilized, resulting in structurally well-defined supramolecular complexes. The hybrid complex could rapidly be assembled, and the kinetics of the formation could be monitored by utilizing the local surface plasmon resonance originating from the gold nanoparticles. We foresee that these findings will aid in designing novel hybrid materials and provide a possible route for the development of functional optoelectronic interfaces for both biomaterials and energy harvesting applications.

\section{INTRODUCTION}

The introduction of chiral optoelectronic functionality into biomacromolecules offers a route to produce hybrid materials for a wide range of bioelectronic applications including electroactive scaffolds for tissue engineering, artificial enzymes, and biosensing. ${ }^{1-4}$ The optoelectronic components can either be conjugated directly to the macromolecular template, ${ }^{5}$ limiting the structural and molecular diversity and increasing the synthetic effort, or be introduced via self-assembly which offers a more scalable and flexible approach. ${ }^{6}$ Conducting polymers (CPs) are routinely used as the optoelectronic component because of their many attractive properties, including excellent light harvesting capability, good photostability, conductivity- and conformation-dependent absorption, and fluorescence. ${ }^{4,7,8}$ However, most CPs reported so far are only compatible with organic solvents because the $\pi$ conjugated backbones are typically functionalized with hydro- phobic side chains. The poor water solubility complicates the processing of the materials as well as their implementation in biological applications. ${ }^{4}$ Conjugated polyelectrolytes (CPEs), on the other hand, contain ionic side chains resulting in good solubility in highly polar media while maintaining excellent optical and electronic function., ${ }^{9,10}$ However, both CPs and CPEs are often generated from random polymerization of different monomeric building blocks resulting in polydisperse materials with randomized positioning of the distinct sidechain functionalities. The lack of chemical precision at the molecular level can limit and restrict the optoelectronic performance of the materials. In contrast, a novel class of conformation-sensitive optical dyes, so-called luminescent-

Received: August 24, 2018

Accepted: October 12, 2018

Published: November 8, 2018 


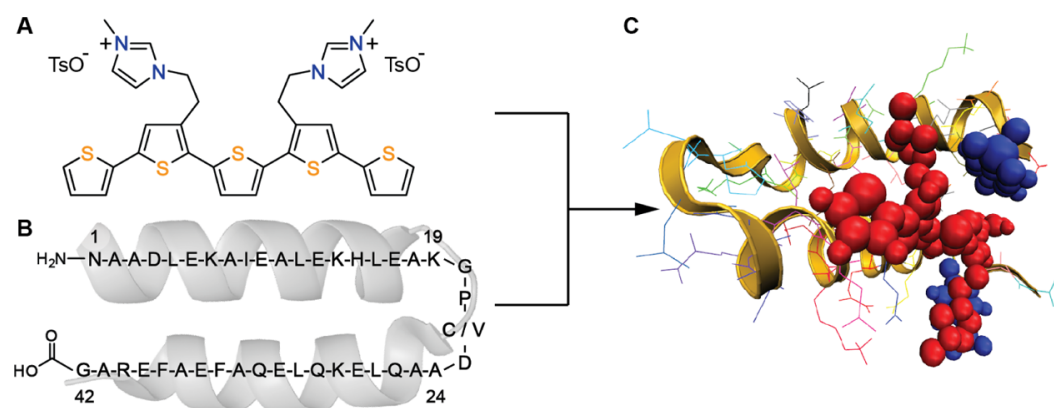

Figure 1. (A) Chemical structure of p-HTMI. (B) Amino acid sequence of JR2EC and JR2E with a cysteine or valine residue at position 22, respectively. (C) Snapshot derived from molecular dynamics (MD) simulations of a one-to-one complex formed between p-HTMI and JR2E. JR2E is represented as a yellow ribbon, and p-HTMI and the counter anion tosylate $\left(\mathrm{TsO}^{-}\right)$are in red and blue, respectively.
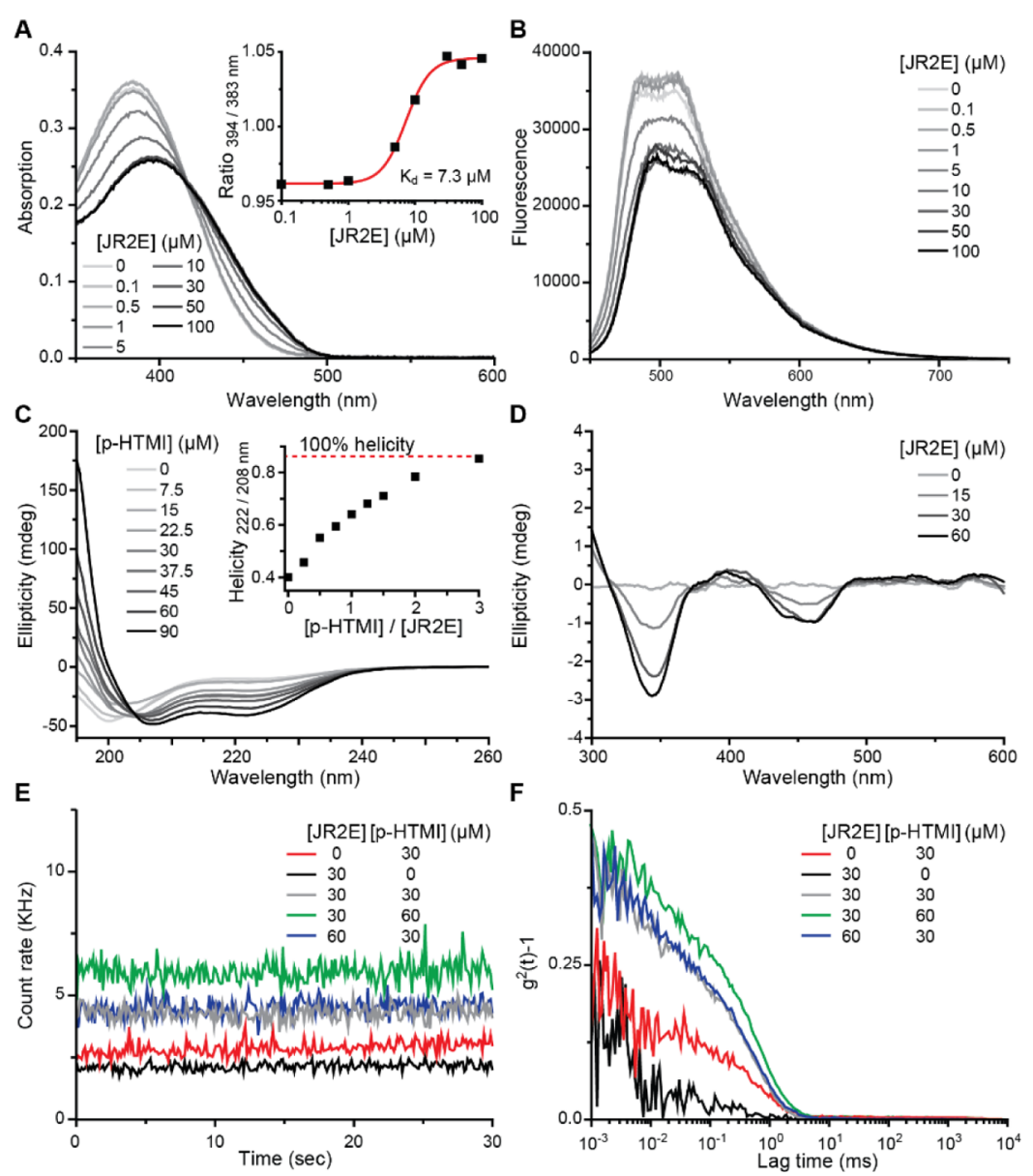

Figure 2. Extinction (A) and emission (B) of p-HTMI $(30 \mu \mathrm{M})$ with increasing concentrations of JR2E $(0-100 \mu \mathrm{M})$. The inset in (A) binding curve derived from the shift in peak position in (A). (C) Circular dichroism (CD) spectra in the UV region of JR2E (30 $\mu \mathrm{M})$ and p-HTMI (0-90 $\mu \mathrm{M})$. The inset in (C) increasing helicity of JR2E due to the addition of p-HTMI with the maximum helicity derived from trifluoroethanol (TFE)treated JR2E indicated as dashed red line. (D) CD spectra in the visible region of p-HTMI $(30 \mu \mathrm{M})$ and JR2E $(0-60 \mu \mathrm{M})$. Scattering intensity (E) and resulting correlation functions (F) from dynamic light scattering (DLS) measurements.

conjugated oligothiophenes (LCOs), combines water solubility with a highly defined chemical structure. ${ }^{11}$ LCOs can be applied as selective and specific ligands for various biomolecular targets and have been employed for investigating numerous different biological phenomena, such as formation of disease-associated protein aggregates and for labeling of distinct cell types. ${ }^{12,13}$ The association of LCOs to proteins and peptides can trigger changes in the LCO backbone conformation resulting in alterations in optical properties which enables identification of the conformational states of the biomolecules. ${ }^{14,15}$ In addition, LCO binding to small biomimetic polypeptides can trigger folding of the peptides leading to induced chirality of the LCO. ${ }^{16}$ Peptides can thus act as structurally well-defined scaffolds for binding of LCOs in a preferred molecular arrangement to generate distinct chirooptical hybrid materials. The possibilities to design and synthesize peptides that can provide both charge complementarity and tailored hydrophobic interactions to match the LCO make peptides ideal candidates as interactional and structural scaffolds. Peptides can also be tethered to inorganic surfaces 
such as gold, silver, platinum, titanium oxide, and zinc sulfide, using various sequence specific interactions, ${ }^{17-22}$ offering a straightforward approach for immobilization and organization of the molecular optoelectronic material on a solid substrate.

Gold nanoparticles (AuNPs) display many attractive physical and chemical properties, such as large optical extinction coefficient in the visible-to-near infrared wavelength range and high chemical stability. These properties are of large relevance in design of optoelectronic materials with tunable properties for applications in energy harvesting, molecular and organic electronics, and biosensing. ${ }^{23-25}$ The optical properties of AuNPs depend on the conditions for the localized surface plasmon resonance (LSPR), which in turn depend on nanoparticle size and shape, interparticle separation, and dielectric properties of the surrounding medium. ${ }^{26}$ The size and shape of the AuNPs can be tuned by a wide range of synthesis strategies, whereas the interparticle separation depends on colloidal stability, which in turn can be modulated by means of the nanoparticle surface chemistry. Changes in colloidal stability triggered by molecular interactions occurring at the particle surface-solvent interface is often employed as a transduction mechanism in colorimetric AuNP-based biosensors. ${ }^{27}$ The LSPR response from immobilized AuNPs is typically less pronounced but can provide direct information about the dielectric properties of the surrounding medium. Immobilized AuNPs can hence be used to probe molecular interaction occurring in the near vicinity of the particle surface in real-time. ${ }^{26}$ AuNPs are thus a good model system for studying molecular interactions and are used in this work to probe the peptide-LCO complex formation at a solid interface.

We have previously studied the self-assembly of anionic LCOs and oligothiophenes with a cationic peptide (JR2K), with focus on how the positioning of electrostatic interactions induce chirality in optically inactive thiophenes. ${ }^{16}$ In this paper, we utilize our previous findings to induce optical activity in a cationic LCO by self-assembling it with a de novo designed negatively charged polypeptide (JR2E) while bound to a substrate. The LCO is an optically inactive pentameric thiophene with two methylated imidazole groups as substituents (p-HTMI) (Figure 1A) and was originally developed as a probe for the detection of cancer associated cells. ${ }^{12,28}$ The anionic polypeptide JR2E is a glutamic-acid-rich 42 residue de novo designed helix-loop-helix polypeptide with a net charge of -5 at neutral $\mathrm{pH}$ (Figure 1B). JR2E is random coil at $\mathrm{pH} 7$ but fold and homodimerize into a four helix bundle at acidic $\mathrm{pH}(\mathrm{pH}<6)$ or in the presence of $\mathrm{mM}$ concentration of $\mathrm{Zn}^{2+}$ ions. $^{29,30}$ The peptide JR2EC is identical to JR2E but has a cysteine residue in position 22 in the loop region instead of a Val, which enables immobilization of JR2EC on AuNPs without significantly effecting dimerization and folding. ${ }^{31}$ In solution, the self-assembly of this anionic peptide and the cationic LCO counterpart (Figure 1C) formed structurally well-defined optically active supramolecular complexes. In addition, we further used the peptide to anchor the LCO onto both suspended and immobilized AuNPs and utilized the changes in interparticle separation as well as the refractive index sensitivity of the AuNPs to study the complex formation on the particle surface. As the possibility to utilize peptidebased scaffolds and molecular self-assembly is an attractive route for the development of hybrid materials with novel optoelectronic properties, we foresee that our findings will aid in the chemical design of novel hybrid materials for organic bioelectronics.

\section{RESULTS AND DISCUSSION}

Optical Characterization of Peptide-LCO Interactions. The association of the peptide JR2E with the LCO pHTMI was verified using several optical techniques. We first investigated the extinction profile of p-HTMI in the presence of JR2E and observed a distinct red shift from 383 to $394 \mathrm{~nm}$ and a decrease in absorption with increasing concentrations of JR2E (Figure 2A). The red shift reached saturation at a JR2Ep-HTMI ratio of $1: 2$, as can be seen in the mole fraction plot (Figure S1). The extinction of p-HTMI is highly dependent on the $\pi-\pi^{*}$ transition region derived from the main chain, and the induced red shift indicates a planarization and an increased effective conjugation length of the thiophene backbone. By using this induced red shift and plotting the ratio of the intensity for the excitation maximum for bound $(393 \mathrm{~nm})$ and unbound $(383 \mathrm{~nm})$ p-HTMI for different concentrations of JR2E, a binding curve could be generated (Figure 2A inset). Fitting of the binding curve to a sigmoidal function (Hill1 function) resulted in an estimated dissociation constant of 7.3 $\mu \mathrm{M}$ which is similar to the previously observed values for binding of anionic LCOs to a cationic peptide. ${ }^{16}$ The emission spectra of the interaction showed a similar trend as the extinction with an increasing red shift and decrease in intensity with increasing concentrations of JR2E, reaching saturation at peptide concentrations at, or above, that of the LCO $(\geq 30 \mu \mathrm{M}$, Figure $2 \mathrm{~B}$ ), further confirming the formation of a complex.

$\mathrm{CD}$ measurements were used to probe for induced structural changes as the complex was formed. As reported previously, JR2E exists as a random coil at neutral $\mathrm{pH}$ with a negative band at 195-200 nm, which is typical for unordered polypeptides. When JR2E was mixed with p-HTMI, the polypeptide adopted a distinct $\alpha$-helical secondary structure with two negative peaks at 208 and $222 \mathrm{~nm}$ in the CD spectra that were more pronounced when the concentration of $\mathrm{p}$ HMTI increased (Figure 2C). To provide a reference point for the maximum amount of folding possible for the peptide, JR2E was treated with TFE as a cosolvent, to enhance and stabilize the secondary structure (Figure S2). ${ }^{32}$ The helicity of the peptide, which can be described by the ratio of the ellipticities at 222 and $208 \mathrm{~nm}$, was comparable to JR2E treated with TFE at a p-HTMI-JR2E ratio of 3:1 (Figure 2C inset). This correlates well with the extinction/emission spectra that showed a saturation of the red shift corresponding to the planarization of the p-HTMI backbone at a similar ratio. Previous studies have shown that JR2E can homodimerize and form four helix bundles at acidic $\mathrm{pH}$ (below $\mathrm{pH}$ 6) when the abundant Glu residues become protonated, which decrease the intermolecular charge repulsion. ${ }^{30}$ Its plausible that homodimerization also occurred due to the association with the cationic p-HTMI because of screening of destabilizing charge interactions, allowing the peptide component to assemble into four helix bundles resulting in a structure with more prominent helicity. We further used $\mathrm{CD}$ in the visible region to characterize p-HTMI which lack optically active substituents and thus failed to produce a CD pattern on its own (Figure 2D). However, in the presence of JR2E, an induced distinct pattern emerged with increasing concentrations of the peptide. The induced chirality seemed to reach saturation at a ratio of two peptides per LCO. The induced CD is most probably derived from the single chain chirality of the conjugated 

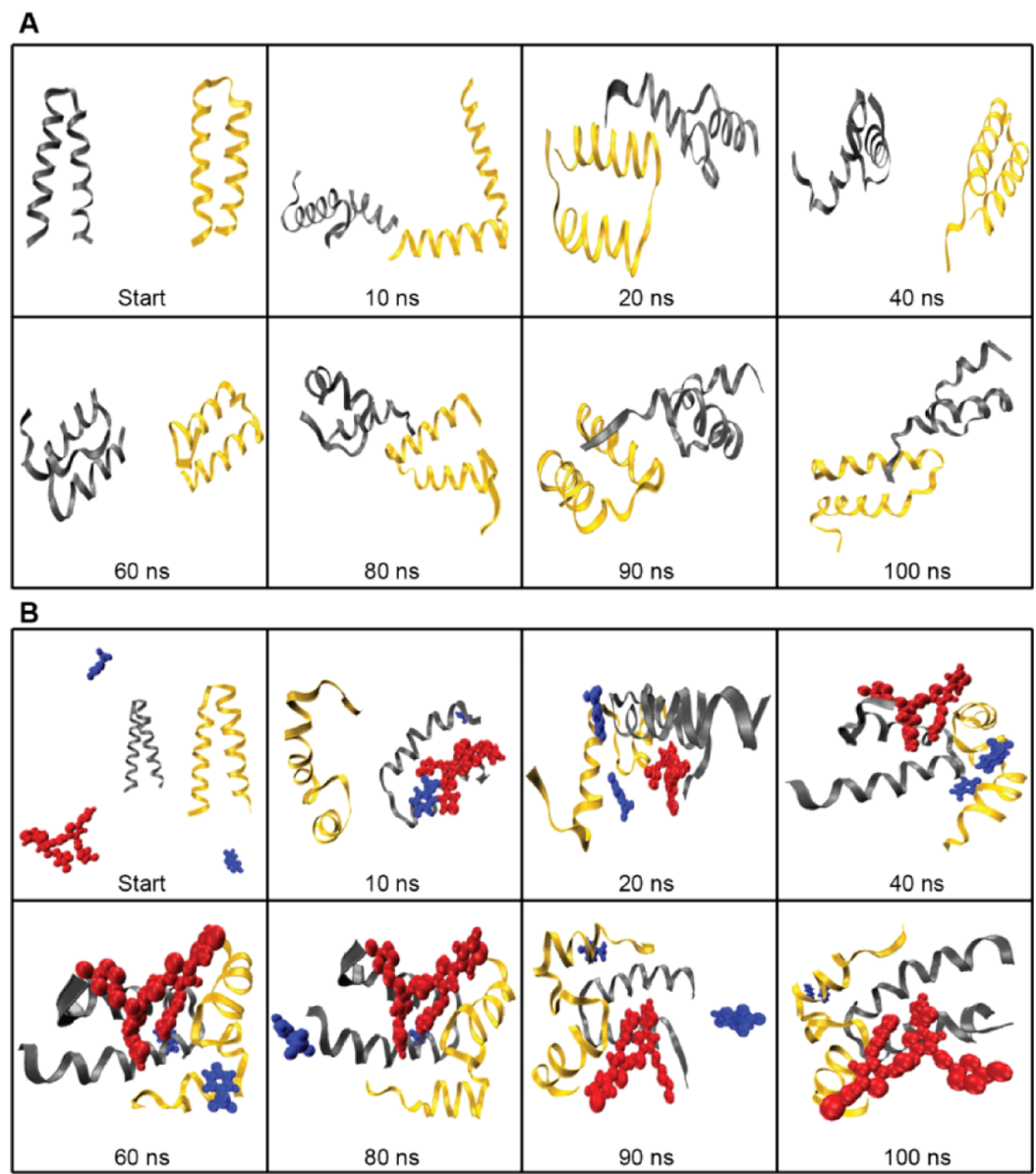

Figure 3. Snapshots of the system of $2 * J R 2 E(A)$ and $2 * J R 2 E+p-H T M I(B)$ at different time points during MD simulation. JR2E is represented in yellow and gray ribbons, and $\mathrm{p}-\mathrm{HTMI}$ and $\mathrm{TsO}^{-}$are in red and blue, respectively.

thiophene backbone as the cationic methylated imidazole groups interact with anionic residues in the peptide affording a chiral template. Thus, the self-assembly of the peptide and the LCOs generated a hybrid complex with unique chiro-optical and structural properties.

Next, DLS was used to investigate the size of the helical chiro-optical complexes formed and dependency of the peptide and LCO stoichiometry (Figure 2E,F). Separately, both the peptide and the LCO produced very low scattering intensities, and the corresponding correlation functions showed no trace of larger assemblies, clearly showing that both constituents are well solubilized in an aqueous environment. When mixed at different ratios, the intensities increased only slightly, where ratios of JR2E-p-HTMI of $1: 1$ and 2:1 produced identical scattering intensities and correlation functions, whereas a 2:1 ratio resulted in slightly higher intensities. Overall, the DLS measurements indicate the formation of well-defined complexes without any large aggregates in an aqueous environment.

Theoretical Calculations of Peptide-LCO Interactions. MD simulations of the peptide and LCO interactions were used to probe the dynamics of the formed complex (Figure 1C) and to get an in depth understanding of the binding site coordination. Binding site analysis suggests that binding occurs mostly because of electrostatic interactions between the cationic methylated imidazole group of p-HTMI and the anionic residues of which the most significant are Glu 6, Glu 10, Glu 28, Glu 32, and Glu 39 (Figure S3). van der
Waals interactions also seem important through nonpolar residues such as Ala 24, Ala 41, Leu 5, and Leu 31 as well as $\pi-\pi$ interactions between the aromatic ring of Phe 35 and the conjugated backbone of p-HTMI (Figure S4). Furthermore, the interaction with the p-HTMI seems to stabilize the folded structure of JR2E as can be seen from the root-mean-square deviation (RMSD) of the polypeptide backbone during the MD simulation (Figure S5). The RMSD values of free JR2E are all higher than the JR2E-p-HTMI complex, illustrating that the binding conformations of polypeptide are less dynamic and the presence of p-HTMI stabilized the folded peptide structure. To investigate the influence of the LCO on homodimerization and the formation of a four-helix bundle between two peptides, two additional systems were modeled consisting of two peptides with (2*JR2E + p-HTMI) and without p-HTMI $(2 * J R 2 E)$. Reviewing the whole trajectories of the simulations using visual molecular dynamics ${ }^{33}$ showed that in the absence of the LCO, the two peptide components failed to form prolonged interactions and only interacted sporadically throughout the simulation (Figure $3 \mathrm{~A}$ ). In the presence of $\mathrm{p}$-HTMI, the homodimer was stabilized, locking the peptides together after initial binding of all components in the system and remaining stable during the residual simulation time (Figure 3B).

To further characterize the influence of p-HTMI on the homodimerization of two monomers of JR2E, the distances between three pairs of residues placed within the two monomers were calculated throughout the simulations (Figure 
$4)$. The residues selected in the first monomer were located at positions 1, 21, and 42 and paired with 21,21 , and 32 in the

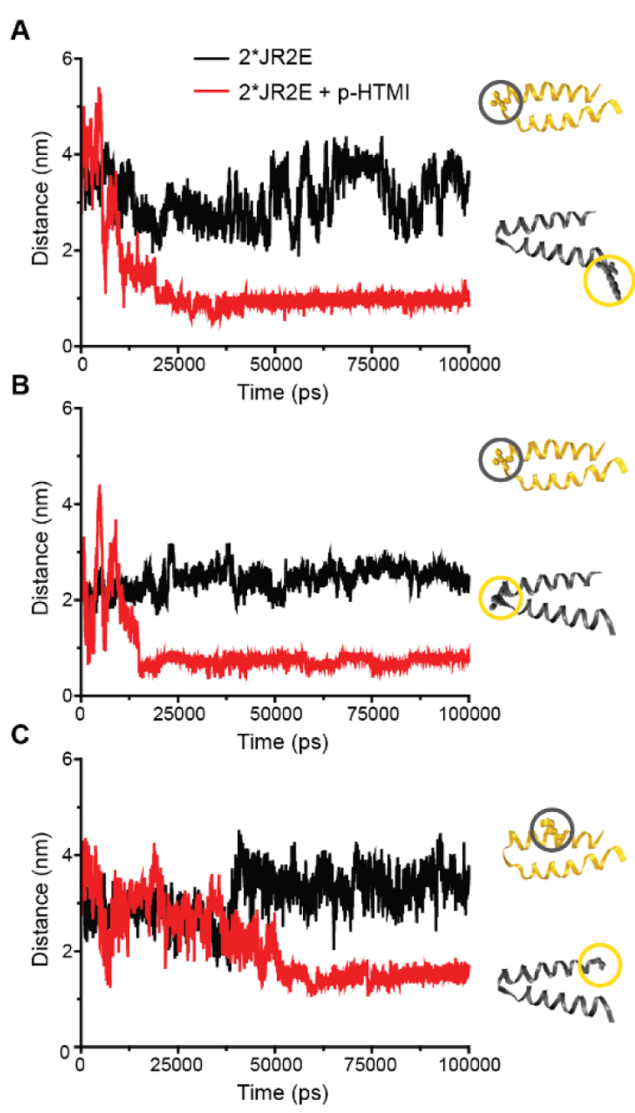

Figure 4. Distance between two residues each located in separate JR2E monomers in two simulated systems, 2*JR2E (black) and 2 *JR2E + p-HTMI (red). (A) Residue number 1 in the first peptide and 21 in the second peptide, (B) residue number 21 in the first peptide and 21 in the second peptide, and (C) residue number 42 in the first peptide and 32 in the second peptide.

second monomer, respectively. The distance between the selected residues was calculated both in the presence and the absence of p-HTMI, and the results show that the presence of the LCO decreased the distance for each of the system trajectories. Both the 1-21 (Figure 4A) and 21-21 (Figure 4B) systems formed stable complexes in the presence of pHTMI already at $\sim 25000$ ps which lasted throughout the simulation. The $42-32$ system formed a stable complex after $\sim 50000 \mathrm{ps}$, which is to be expected because the selected residues are located near the more dynamic $\mathrm{N}$-terminals of the peptide monomers (Figure 4C).

Peptide-LCO Complex Formation on Colloidal AuNPs. Both optical characterization and MD simulations thus show that the peptide indeed can function as a scaffold, orientating and inducing optical activity in the LCO. In addition, the peptide and LCO and the final supramolecular hybrid material display excellent solubility in an aqueous environment without the formation of large aggregates which is promising for further development of highly defined biocompatible optoelectronic devices. We further went on to investigate if the peptide could be used as a structural scaffold to anchor the LCO onto a solid substrate. AuNPs were used as a model system because of the possibilities to exploit the nanoplasmonic properties of the nanoparticles to investigate the formation of the dynamic surface bound complex. To immobilize the peptide to AuNPs, a second peptide (JR2EC), that is identical to JR2E in all regards except that it contains a cysteine residue in the loop region was synthesized. The JR2EC-functionalized particles (JR2EC-AuNPs) have an exceptional colloidal stability both because of the high negative net charge of the peptide resulting in electrostatic repulsion between particles according to the DLVO theory ${ }^{34}$ and the steric stabilization afforded by the peptide coating. The colloidal stability can be tuned by $\mathrm{pH}$ or addition of metal cations, as reported previously. ${ }^{29,30}$ Reversible nanoparticle self-assembly can thus be triggered as a result of folding and homodimerization between peptides situated on neighboring AuNPs.

By exposing the peptide-functionalized AuNPs to p-HTMI, the immobilized peptide can be used as a scaffold for anchoring the LCO to the particle surface in a chemically and structurally defined fashion. Because the association of the LCO to the immobilized peptide will affect both the peptide net charge and the folding of the peptide and promote homodimerization, the colloidal stability will be affected (Figure 5A). The interaction can thus be monitored by assessing changes in the LSPR response from the particles. Before investigating the effect of p-HTMI on JR2EC-AuNPs, we concluded that no overlap in the extinction spectrum of the LSPR band deriving from the AuNPs and the extinction from the LCO occurred (Figure S6). When exposing JR2EC-AuNPs to p-HTMI $(3 \mu \mathrm{M})$, particle aggregation occurred and the LSPR peak maximum $\left(\lambda_{\max }\right)$ was massively red shifted from 524 to $590 \mathrm{~nm}$, reaching a saturation after $20 \mathrm{~min}$ (Figure 5B). The large red shift occurs due to plasmon coupling as the particle plasmons start to overlap when the interparticle distance is decreased. ${ }^{35}$ The shift in $\lambda_{\max }\left(\Delta \lambda_{\max }\right)$ induced by the LCO was highly dependent on the p-HTMI concentration (Figure 5C), where concentrations of LCO above $2 \mu \mathrm{M}$ caused a rapid aggregation of the JR2EC-AuNPs. By plotting the $\Delta \lambda_{\max }$ for the different concentrations of LCO after reaching saturation (20 $\mathrm{min})$, a clear dose responsive curve for the LCO-induced aggregation was found within a narrow concentration span of $1.75-4 \mu \mathrm{M}$ p-HTMI (Figure 5D). Fitting the data to a monophasic hill equation, the concentration of LCO required for producing a $50 \%$ LSPR response $\left(\Delta \lambda_{50 \%}\right)$ could be calculated to be $2.5 \mu \mathrm{M}$. This value correlates well with the calculated $K_{\mathrm{d}}$ for the complex formation derived from extinction data $(7.3 \mu \mathrm{M})$. Thus, the peptide-LCO complex could also be afforded when the peptide was attached to a solid support. Furthermore, transmission electron micrographs of JR2EC-AuNPs treated with p-HTMI showed large aggregates of particles with a distinct spacing between individual particles (Figure 5E), which is to be expected as the peptide-LCO complexes formed in between the AuNPs have a low electron density and will thus not be visible in the TEM.

Peptide-LCO Complex Formation on Immobilized AuNPs. To further study the peptide-LCO complex formation on a particle surface using LSPR but without affecting the colloidal stability of the AuNPs, thus avoiding plasmon coupling and homodimerization of the peptide, the AuNPs were immobilized on a substrate (Figure 6A). The negatively charged AuNPs were electrostatically immobilized using a layer-by-layer assembly of polyelectrolytes with a positively charged polymer as the top layer directly in a microwell plate. $^{36}$ The AuNPs were subsequently function- 

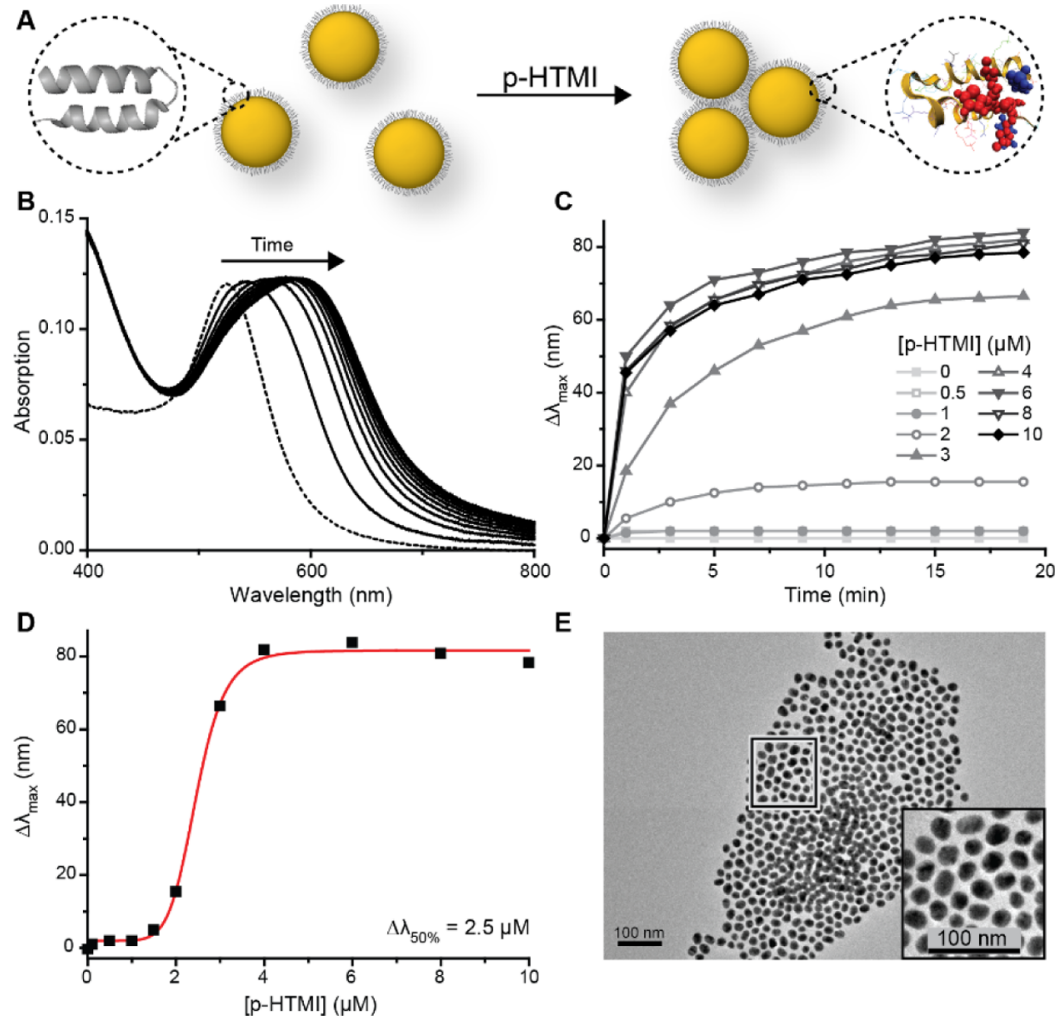

Figure 5. (A) Schematic illustration of the JR2EC-AuNP assembly due to complex formation of JR2EC-p-HTMI on the particle surface used in (B-E). (B) UV-vis spectra of JR2EC-AuNPs (dashed line) and after treatment with p-HTMI ( $3 \mu \mathrm{M}$, solid line) recorded over 20 min. (C) LSPR peak shifts over time with increasing concentration of p-HTMI. (D) LSPR peak shifts after 20 min as a function of p-HTMI concentration. (E) Transmission electron microscopy (TEM) micrographs of JR2EC-AuNPs treated with p-HTMI.

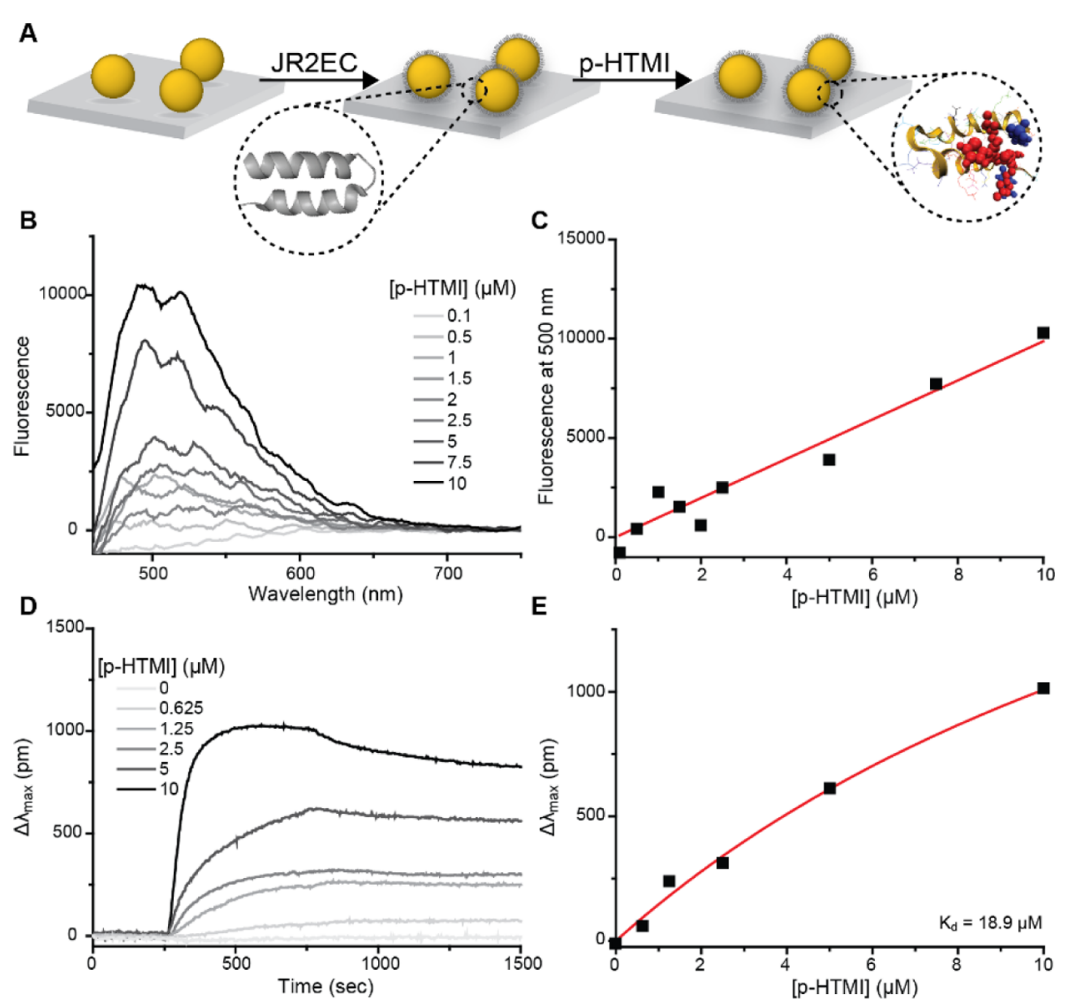

Figure 6. (A) Schematic illustration of functionalization of immobilized AuNPs with JR2EC and complex formation of JR2EC-p-HTMI on the particle surface used in (B-E). (B) Fluorescence of p-HTMI after complex formation on the surface of JR2EC-AuNPs under static conditions with subsequence wash. (C) Concentration-dependent fluorescence at $500 \mathrm{~nm}$ derived from (C). (D) Real-time LSPR in a flow setup with increasing concentrations of p-HTMI $(0-10 \mu \mathrm{M})$. (E) Peak shifts at $750 \mathrm{~s}$ derived from (D) fitted to a steady-state affinity model. 
alized with JR2EC, and the interaction with p-HTMI was probed using the LSPR shifts that solely depend on interactions occurring at the solution-particle interface. In static (nonflow) LSPR measurements with increasing concentrations of p-HTMI, a dose-dependent shift in the LSPR peak position could be detected (Figure S7). Washing the immobilized JR2EC-AuNPs with buffer did not alter the LSPR response, confirming the firm attachment of the LCO. We further investigated if the emission from the attached LCO could be detected. Prior to washing the wells with immobilized JR2EC-AuNPs with buffer, a decreased emission from pHTMI could be detected as the complex formation occurred at the particle-solvent interface (Figure S8), which reduced the concentration of free LCO in the buffer. This is consistent with the observation that the JR2E-p-HTMI complex formation resulted in the decreased emission intensity (Figure $2 \mathrm{~B}$ ). It is also plausible that the decrease was not due to AuNP-coupled quenching as saturation could be observed for high concentrations of LCO which can be attributed to complexation to all available peptides. The emission after rinsing off unbound LCO could also be detected (Figure 6B), despite the low surface LCO concentration which is due to the immobilization of JR2EC-AuNPs on to a planar 2D substrate with a particle surface coverage of approximately $10 \%$. A clear correlation between emission and LCO concentration could be seen when plotting the emission at $500 \mathrm{~nm}$ as a function of the LCO concentration (Figure 6C). We further evaluated if the kinetics of the association of p-HTMI to JR2EC could be studied using the same LSPR-based setup but coupled to a flow system. AuNP immobilization was performed on glass cover slips modified by the layer-by-layer assembly of polyelectrolytes, and the AuNPs were subsequently functionalized with JR2EC as described previously. ${ }^{37}$ LSPR measurements in a $100 \mu \mathrm{L} / \mathrm{min}$ flow with different concentrations of $\mathrm{p}$ HTMI resulted in real-time sensorgrams for the interaction (Figure 6D). By plotting the LSPR response at the end of the injection $(750 \mathrm{~s})$ and fitting the data to a steady-state affinity model, a dissociation constant for the interaction was calculated to be $18.9 \mu \mathrm{M}$ (Figure 6E). The dissociation constant for the association of p-HTMI to immobilized JR2EC formation was thus about a 2 factor higher than that for the association in solution, which is surprisingly similar considering the constrains associated with a surface bound complexation process.

\section{CONCLUSIONS}

In conclusion, we have shown that a hybrid material with distinct chiro-optical properties and architectural arrangement could be generated via self-assembly of an anionic peptide and a cationic chemical-defined pentameric thiophene. Selfassembly of the hybrid material induces structural changes in the peptide, from random coil to a distinct helical conformation, and induces optical activity in the $\pi-\pi^{*}$ transition region for the pentameric thiophene. Thus, the hybrid material exhibits unique structural and chiro-optical properties that are afforded because of the self-assembly of the peptide and the optoelectronic element. Additionally, both the individual constituents and the final hybrid material display excellent solubility in an aqueous environment without the formation of large aggregates which is promising for further development of highly defined optoelectronic structures and devices. Theoretical calculations confirm the experimentally observed induced structural changes in the peptide compo- nent, as well as the electrostatic and hydrophobic interactions contributing to the overall stability of the complex. The peptide-LCO complex formation could also be afforded on a solid support showing the plausibility of using a peptide as a scaffold to orientate and self-assemble optoelectronic functionality to the surface of a substrate. Gold nanoparticles were used as a model system to enable LSPR-based monitoring of the self-assembly process using both immobilized and dispersed nanoparticles. Equilibrium was reached within minutes, and the slow dissociation indicates formation of robust complexes. Because of the large number of possible strategies to immobilize peptides on solid substrates, ${ }^{38}$ including the possibility to use various metal and oxide binding peptide sequences, ${ }^{17-22}$ this assembly strategy is not only limited to gold but also opens up for use of other and potentially more relevant substrates. This straightforward approach for generating well-defined molecular structures on a solid support that can be used as molecular optoelectronic antennas allows for intriguing opportunities to further develop biocompatible optoelectronic devices, biosensors, and strategies for energy harvesting and artificial enzymes.

\section{EXPERIMENTAL SECTION}

Materials. The peptides JR2E ( $\mathrm{H}_{2} \mathrm{~N}-\mathrm{NAADLEKAIEA-}$ LEKHLEAKGPVDAAQLEKQLEQAFEAFERAG-COOH) and JR2EC $\left(\mathrm{H}_{2} \mathrm{~N}-\mathrm{NAADLEKAIEALEKHLEAKGPCDAAQ-}\right.$ LEKQLEQAFEAFERAG-COOH) were synthesized as described previously. ${ }^{29}$ The tosylate $\left(\mathrm{TsO}^{-}\right)$salt of p-HTMI was synthesized as described earlier. ${ }^{12}$

All measurements were performed in bis-tris buffer (30 $\mathrm{mM}, \mathrm{pH}$ 7.0) expect $\mathrm{CD}$ measurements which were performed in phosphate buffer (10 mM, pH 7.0).

AuNPs for Solution-Based Assays. The polypeptidefunctionalized gold nanoparticles with an average diameter of $20 \mathrm{~nm}$ (Cline Scientific AB, Sweden) were prepared by an addition of $15 \mu \mathrm{L}$ of a $1 \mathrm{mM}$ JR2EC solution $(10 \mathrm{mM}$ sodium citrate, $\mathrm{pH} \mathrm{6)}$ to $1.485 \mathrm{~mL}$ of the gold colloidal solution. The mixture was incubated overnight at $4{ }^{\circ} \mathrm{C}$ and then repeatedly centrifuged in order to remove the excess unbound peptides and exchange the buffer with fresh bis-tris buffer $(30 \mathrm{mM}, \mathrm{pH}$ 7.0), until the concentration of free JR2EC was negligible (less than $5 \mathrm{pM}$ ). The final concentration of JR2EC-AuNPs was estimated to be $7.7 \mathrm{nM}$ using the absorbance at $525 \mathrm{~nm}$ and an extinction coefficient of $1.57 \times 10^{9} \mathrm{M}^{-1} \mathrm{~cm}^{-1}$. 39

Optical Characterization. The extinction and emission spectra were collected using a Tecan Infinite M1000 Pro microplate reader from Tecan Männedorf (Switzerland). The concentration of p-HTMI was set to $30 \mu \mathrm{M}$, and the concentration of JR2E was varied between 0 and $100 \mu \mathrm{M}$. Samples were incubated for $10 \mathrm{~min}$ before measurements. Adsorption spectra were collected in the range 350-600 nm and background subtracted. Emission spectra were collected in the range $450-750 \mathrm{~nm}$ with an excitation wavelength of 380 nm.

CD Spectroscopy. CD measurements were performed on a Chirascan spectropolarimeter from Applied Photophysics (UK). CD measurements in the near UV region were performed in a $0.1 \mathrm{~mm}$ cuvette between 195 and $260 \mathrm{~nm}$ at room temperature. The concentration of JR2E was fixed at 30 $\mu \mathrm{M}$, and the concentration $\mathrm{p}-\mathrm{HTMI}$ was varied between 0 and $90 \mu \mathrm{M}$. To provide a reference spectrum for the maximum amount of peptide folding, JR2E $(30 \mu \mathrm{M})$ was treated with $50 \%$ TFE in $\mathrm{ddH}_{2} \mathrm{O}$ and incubated for $10 \mathrm{~min}$ prior to 
measurement. Measurements in the visible region were performed in a $1 \mathrm{~mm}$ cuvette between 300 and $600 \mathrm{~nm}$ at room temperature. The concentration of $\mathrm{p}$-HTMI was fixed at $30 \mu \mathrm{M}$, and the concentration JR2E was varied between 0 and $60 \mu \mathrm{M}$. Samples were incubated for $10 \mathrm{~min}$ before measurements, and each spectrum was collected as an average of three scans and background subtracted.

Dynamic Light Scattering. DLS measurements of JR2E and p-HTMI separate and in combination were performed on an ALV/DLS/SLS-5022F system from ALV GmbH (Germany) using a $\mathrm{HeNe}$ laser at $632.8 \mathrm{~nm}$. JR2E $(0-60 \mu \mathrm{M})$ and buffers were filtered using a polyvinylidene fluoride filter prior to the addition of p-HTMI $(0-60 \mu \mathrm{M})$. Samples were incubated for $10 \mathrm{~min}$ and tempered to $22{ }^{\circ} \mathrm{C}$ and all measurements were repeated 10 times and the collected data were averaged.

Molecular Dynamics Simulation. The structure of the 42 reside helix-loop-helix polypeptide JR2E was prepared using the four-helix bundle protein (PDB code: $1 \mathrm{MFT})^{40}$ utilizing Discovery Studio software ${ }^{41}$ in which the four-helix bundle was cut to one helix loop helix and the amino acids of JR2E replaced the amino acids of PDB.

The structure of LCO, both cation, p-HTMI, and anion, OTs (4-methylbenzenesulfonate), was drawn in Gauss view and optimized with the B3LYP method and 6-31G* basis set using the program Gaussian $09,{ }^{42}$ and the atomic charges were calculated by fitting the molecular electrostatic potential of the electronic density using the CHELPG procedure, ${ }^{43}$ then using PRODRG server to generate topology files for the LCO. ${ }^{44}$

The simulations were carried out using GROMACS package 5.1.2. ${ }^{45}$ The GROMOS $9643 \mathrm{al}$ force field and the simple point charge model of water were employed. ${ }^{46}$ All simulations were performed in a cubic simulation box with the dimensions of $7 \times 7 \times 7 \mathrm{~nm}^{3}$. The energy of each system was minimized using the steepest descent algorithm. The simulations were done in canonical ensemble NVT at a temperature of $300 \mathrm{~K}$ using a v-rescale thermostat, ${ }^{47}$ and the pressure was coupled to a Parrinello-Rahman barostat at 1 atm. ${ }^{48}$ The simulations were carried out by the leap-frog integration algorithm at a time step of 2 fs. The LINCS algorithm was used to constrain bond lengths, ${ }^{49}$ and the SETTLE algorithm was applied to constrain water bond lengths. ${ }^{50}$ Particle mesh Ewald was used for long-range electrostatics. ${ }^{33}$ The cutoff distance of $1.0 \mathrm{~nm}$ was used for both electrostatic and van der Waals interactions. In addition, in all directions, the periodic boundary conditions were applied. Then, the simulation was performed in $100 \mathrm{~ns}$. The graphical representation of MD results was produced using the VMD program. ${ }^{51}$

Optical Characterization of JR2EC-AuNPs. Absorption spectra of the JR2EC-AuNPs were measured on a Shimadzu UV-2450 spectrophotometer (Japan). JR2EC-AuNPs (77 pM) were subjected to p-HTMI $(0-10 \mu \mathrm{M})$, and the LSPR peak was monitored for $20 \mathrm{~min}$.

Transmission Electron Microscopy. TEM micrographs were acquired using a Tecnai G2 F20 Ultra-Twin microscope from Fei (USA) operating at $200 \mathrm{kV}$. Grids were prepared by applying one drop of p-HTMI-treated JR2EC-AuNPs onto a carbon-coated copper grid, and excess water was gently wiped off with filter paper, and the grid was air-dried before analysis.

Static LSPR Detection. A transparent 96-well plate with flat, round bottoms (Nunc, Thermo Fischer Scientific) was used as a substrate and prepared by plasma treatment (Diener Pico, Diener electronics, 0.8 mbar, 240 s, $200 \mathrm{~W}, 40 \mathrm{kHz}$ generator, gas used: oxygen) for cleaning and creating a negatively charged surface. After plasma treatment, gold nanoparticles (BBI, $\varnothing=50 \mathrm{~nm}$ ) were immobilized into the wells using polyelectrolytes. Polyelectrolyte solutions of polyethylenimine (PEI, $M_{\mathrm{w}} 750$ 000, Sigma-Aldrich), polystyrene sulfonate (PSS, $M_{\mathrm{w}} 75$ 000, Sigma-Aldrich), and polyallylamine hydrochloride $\left(\mathrm{PAH}, M_{\mathrm{w}} 56000\right.$, SigmaAldrich) were prepared with a concentration of $2 \mathrm{mg} / \mathrm{mL}$ in $0.5 \mathrm{M} \mathrm{NaCl}$ (Sigma-Aldrich) aqueous solutions. The polyelectrolyte solution $(100 \mu \mathrm{L})$ was added to each well for $10 \mathrm{~min}$ on the order $\mathrm{PEI} / \mathrm{PSS} / \mathrm{PAH} / \mathrm{PSS} / \mathrm{PAH}$ with a thorough rinsing of $\mathrm{ddH}_{2} \mathrm{O}$ between each deposition. Finally, $100 \mu \mathrm{L}$ of a gold nanoparticle suspension (BBI, $\varnothing=50 \mathrm{~nm}$ ) was added to the wells for $4 \mathrm{~h}$, followed by rinsing with $\mathrm{ddH}_{2} \mathrm{O}$. Prior to the LSPR measurements, the immobilized gold nanoparticles were functionalized by adding $50 \mu \mathrm{L} /$ well of $10 \mu \mathrm{M}$ JR2EC in $\mathrm{ddH}_{2} \mathrm{O}$ for $2 \mathrm{~h}$ followed by rinsing with $\mathrm{ddH}_{2} \mathrm{O}$. Data were obtained by monitoring the plasmon peak position after incubation of $50 \mu \mathrm{L} /$ well of different concentrations of p-HTMI for $30 \mathrm{~min}$ onto the peptidefunctionalized nanoparticle substrates. All samples were prepared in three replicates.

Absorbance scan was measured at $400-800 \mathrm{~nm}$ by a Tecan Infinite M1000 Pro microplate reader. Fluorescence spectra were collected in the range $450-750 \mathrm{~nm}$ with an excitation wavelength of $380 \mathrm{~nm}$. The polyelectrolyte layer without AuNP was used as a control and data subtracted prior to analysis. Absorbance data were fitted (9th degree polynomial) and analyzed for peak position with Matlab (MATLAB and Statistics Toolbox Release 2017a, The MathWorks, Inc., Natick, Massachusetts, United States).

Real-Time LSPR Detection. Real-time LSPR measurements were performed using a custom-made setup comprising a halogen light source (Ocean Optics HL-2000), a USBspectrophotometer (Ocean Optics QE65 Pro), an injection port valve (VICI Valco), a fluidic pump (Shimadzu LC-20AD), and an in-house fabricated flow cell. Real-time tracking of the plasmon peak position was performed using an in-house developed LabView software. Round glass cover slips (Fisher Scientific, $d=13 \mathrm{~mm}$ ) were used as substrates and prepared by initial cleaning in a solution containing a 5:1:1 mixture by volume of $\mathrm{ddH}_{2} \mathrm{O}, 30 \%$ hydrogen peroxide (Merck $\mathrm{KGaA}$ ), and $25 \%$ ammonia (Merck $\mathrm{KGaA}$ ) for $5 \mathrm{~min}$ at $85{ }^{\circ} \mathrm{C}$ and thoroughly rinsed with $\mathrm{ddH}_{2} \mathrm{O}$. Gold nanoparticles were immobilized onto the substrates using polyelectrolytes. Polyelectrolyte solutions of PEI $\left(M_{\mathrm{w}} 750000\right.$, Sigma-Aldrich), PSS $\left(M_{\mathrm{w}} 75000\right.$, Sigma-Aldrich), and PAH $\left(M_{\mathrm{w}} 56000\right.$, Sigma-Aldrich) were prepared with a concentration of $2 \mathrm{mg} /$ $\mathrm{mL}$ in $0.5 \mathrm{M} \mathrm{NaCl}$ (Sigma-Aldrich) aqueous solutions. The polyelectrolyte solution $(100 \mu \mathrm{L})$ was added to the glass substrates for $15 \mathrm{~min}$ on the order PEI/PSS/PAH/PSS/PAH with a thorough rinsing of $\mathrm{ddH}_{2} \mathrm{O}$ between each deposition. Finally, $200 \mu \mathrm{L}$ of a gold nanoparticle suspension (BBI, $d=50$ $\mathrm{nm}$ ) was added to the substrates for $4 \mathrm{~h}$, followed by rinsing with $\mathrm{ddH}_{2} \mathrm{O}$. Prior to the real-time LSPR tests, the immobilized gold nanoparticles were functionalized by adding $200 \mu \mathrm{L}$ of $10 \mu \mathrm{M} \mathrm{JR} 2 \mathrm{EC}$ in water for $2 \mathrm{~h}$, followed by rinsing with $\mathrm{ddH}_{2} \mathrm{O}$. Kinetic data were obtained by monitoring the plasmon peak position during injection of $500 \mu \mathrm{L}$ of different concentrations of p-HTMI over a peptide-functionalized nanoparticle substrate with a flow rate of $100 \mu \mathrm{L} / \mathrm{min}$. Data were evaluated using BIAevaluation software. 


\section{ASSOCIATED CONTENT}

\section{S Supporting Information}

The Supporting Information is available free of charge on the ACS Publications website at DOI: 10.1021/acsomega. 8 b02153.

Mole fraction plot, CD spectrum of JR2E in TFE, binding site analysis, MD simulation of RMSD for complex formation, UV-vis of AuNPs and LCO, and LSPR peak shifts and fluorescence in static setup (PDF)

\section{AUTHOR INFORMATION}

\section{Corresponding Author \\ *E-mail: robert.selegard@liu.se. \\ ORCID}

Daniel Aili: 0000-0002-7001-9415

Mohammad R. Housaindokht: 0000-0002-5428-2512

K. Peter R. Nilsson: 0000-0002-5582-140X

Robert Selegård: 0000-0002-1781-1489

\section{Notes}

The authors declare no competing financial interest.

\section{ACKNOWLEDGMENTS}

This work was financed by the Knut and Alice Wallenberg Foundation (Z.R., D.A., E.M., A.S., and R.S.), the Swedish Foundation for Strategic Research (Z.R., D.A., E.M., A.S., M.B., K.P.R.N., and R.S.), and the Swedish Government Strategic Research Area in Materials Science on Functional Materials at Linköping University (Faculty grant SFO Mat LiU no. 2009 00971).

\section{REFERENCES}

(1) Guo, B.; Ma, P. X. Conducting Polymers for Tissue Engineering. Biomacromolecules 2018, 19, 1764-1782.

(2) Pal, R. K.; Farghaly, A. A.; Wang, C.; Collinson, M. M.; Kundu, S. C.; Yadavalli, V. K. Conducting polymer-silk biocomposites for flexible and biodegradable electrochemical sensors. Biosens. Bioelectron. 2016, 81, 294-302.

(3) Gauthier, M. A.; Klok, H.-A. Polymer-protein conjugates: an enzymatic activity perspective. Polym. Chem. 2010, 1, 1352-1373.

(4) Inal, S.; Rivnay, J.; Suiu, A.-O.; Malliaras, G. G.; McCulloch, I. Conjugated Polymers in Bioelectronics. Acc. Chem. Res. 2018, 51, 1368-1376.

(5) Schillinger, E.-K.; Mena-Osteritz, E.; Hentschel, J.; Börner, H. G.; Bäuerle, P. Oligothiophene Versus $\beta$-Sheet Peptide: Synthesis and Self-Assembly of an Organic Semiconductor-Peptide Hybrid. Adv. Mater. 2009, 21, 1562-1567.

(6) Barbarella, G.; Di Maria, F. Supramolecular Oligothiophene Microfibers Spontaneously Assembled on Surfaces or Coassembled with Proteins inside Live Cells. Acc. Chem. Res. 2015, 48, 2230-2241.

(7) Arnaboldi, S.; Grecchi, S.; Magni, M.; Mussini, P. Electroactive chiral oligo- and polymer layers for electrochemical enantiorecognition. Curr. Opin. Electrochem. 2018, 7, 188-199.

(8) Das, T. K.; Prusty, S. Review on Conducting Polymers and Their Applications. Polym. Plast. Technol. Eng. 2012, 51, 1487-1500.

(9) Jiang, H.; Taranekar, P.; Reynolds, J. R.; Schanze, K. S. Conjugated Polyelectrolytes: Synthesis, Photophysics, and Applications. Angew. Chem., Int. Ed. 2009, 48, 4300-4316.

(10) Lee, W.; Seo, J. H.; Woo, H. Y. Conjugated polyelectrolytes: A new class of semiconducting material for organic electronic devices. Polymer 2013, 54, 5104-5121.

(11) Åslund, A.; Nilsson, K. P. R.; Konradsson, P. Fluorescent oligo and poly-thiophenes and their utilization for recording biological events of diverse origin-when organic chemistry meets biology. $J$. Chem. Biol. 2009, 2, 161-175.
(12) Cieślar-Pobuda, A.; Bäck, M.; Magnusson, K.; Jain, M. V.; Rafat, M.; Ghavami, S.; Nilsson, K. P. R.; Łos, M. J. Cell type related differences in staining with pentameric thiophene derivatives. Cytometry Part A 2014, 85, 628-635.

(13) Löffler, S.; Melican, K.; Nilsson, K. P. R.; Richter-Dahlfors, A. Organic bioelectronics in medicine. J. Intern. Med. 2017, 282, 24-36.

(14) Psonka-Antonczyk, K. M.; Hammarström, P.; Johansson, L. B. G.; Lindgren, M.; Stokke, B. T.; Nilsson, K. P. R.; Nyström, S. Nanoscale structure and spectroscopic probing of $\mathrm{A} \beta 1-40$ fibril bundle formation. Front. Chem. 2016, 4, 44.

(15) Wigenius, J.; Björk, P.; Hamedi, M.; Aili, D. Supramolecular Assembly of Designed $\alpha$-Helical Polypeptide-Based Nanostructures and Luminescent Conjugated Polyelectrolytes. Macromol. Biosci. 2010, 10, 836-841.

(16) Selegård, R.; Rouhbakhsh, Z.; Shirani, H.; Johansson, L. B. G.; Norman, P.; Linares, M.; Aili, D.; Nilsson, K. P. R. Distinct Electrostatic Interactions Govern the Chiro-Optical Properties and Architectural Arrangement of Peptide-Oligothiophene Hybrid Materials. Macromolecules 2017, 50, 7102-7110.

(17) Pacardo, D. B.; Sethi, M.; Jones, S. E.; Naik, R. R.; Knecht, M. R. Biomimetic Synthesis of Pd Nanocatalysts for the Stille Coupling Reaction. ACS Nano 2009, 3, 1288-1296.

(18) Lévy, R. Peptide-Capped Gold Nanoparticles: Towards Artificial Proteins. ChemBioChem 2006, 7, 1141-1145.

(19) Dickerson, M. B.; Jones, S. E.; Cai, Y.; Ahmad, G.; Naik, R. R.; Kröger, N.; Sandhage, K. H. Identification and Design of Peptides for the Rapid, High-Yield Formation of Nanoparticulate TiO2from Aqueous Solutions at Room Temperature. Chem. Mater. 2008, 20, $1578-1584$

(20) Naik, R. R.; Stringer, S. J.; Agarwal, G.; Jones, S. E.; Stone, M. $\mathrm{O}$. Biomimetic synthesis and patterning of silver nanoparticles. Nat. Mater. 2002, 1, 169-172.

(21) Lee, S.-W.; Mao, C.; Flynn, C. E.; Belcher, A. M. Ordering of Quantum Dots Using Genetically Engineered Viruses. Science 2002, 296, 892-895.

(22) Sarikaya, M.; Tamerler, C.; Jen, A. K.-Y.; Schulten, K.; Baneyx, F. Molecular biomimetics: nanotechnology through biology. Nat. Mater. 2003, 2, 577-585.

(23) Ozbay, E. Plasmonics: Merging Photonics and Electronics at Nanoscale Dimensions. Science 2006, 311, 189-193.

(24) Edel, J. B.; Kornyshev, A. A.; Urbakh, M. Self-assembly of nanoparticle arrays for use as mirrors, sensors, and antennas. ACS Nano 2013, 7, 9526-9532.

(25) Mo, L.; Li, J.; Liu, Q.; Qiu, L.; Tan, W. Nucleic acidfunctionalized transition metal nanosheets for biosensing applications. Biosens. Bioelectron. 2017, 89, 201-211.

(26) Willets, K. A.; Van Duyne, R. P. Localized Surface Plasmon Resonance Spectroscopy and Sensing. Annu. Rev. Phys. Chem. 2007, 58, 267-297.

(27) Wilson, R. The use of gold nanoparticles in diagnostics and detection. Chem. Soc. Rev. 2008, 37, 2028-2045.

(28) Magnusson, K.; Appelqvist, H.; Cieślar-Pobuda, A.; Bäck, M.; Kågedal, B.; Jonasson, J. A.; Los, M. J.; Nilsson, K. P. R. An imidazole functionalized pentameric thiophene displays different staining patterns in normal and malignant cells. Front. Chem. 2015, 3, 58.

(29) Aili, D.; Enander, K.; Rydberg, J.; Nesterenko, I.; Björefors, F.; Baltzer, L.; Liedberg, B. Folding Induced Assembly of Polypeptide Decorated Gold Nanoparticles. J. Am. Chem. Soc. 2008, 130, 57805788.

(30) Aili, D.; Enander, K.; Rydberg, J.; Lundström, I.; Baltzer, L.; Liedberg, B. Aggregation-Induced Folding of a De Novo Designed Polypeptide Immobilized on Gold Nanoparticles. J. Am. Chem. Soc. 2006, 128, 2194-2195.

(31) Aili, D.; Enander, K.; Baltzer, L.; Liedberg, B. Assembly of Polypeptide-Functionalized Gold Nanoparticles through a Heteroassociation- and Folding-Dependent Bridging. Nano Lett. 2008, 8, $2473-2478$ 
(32) Buck, M. Trifluoroethanol and colleagues: cosolvents come of age. Recent studies with peptides and proteins. Q. Rev. Biophys. 1998, $31,297-355$.

(33) Humphrey, W.; Dalke, A.; Schulten, K. VMD: visual molecular dynamics. J. Mol. Graphics 1996, 14, 33-38.

(34) Ghosh, S. K.; Pal, T. Interparticle Coupling Effect on the Surface Plasmon Resonance of Gold Nanoparticles: From Theory to Applications. Chem. Rev. 2007, 107, 4797-4862.

(35) Chen, T.; Pourmand, M.; Feizpour, A.; Cushman, B.; Reinhard, B. M. Tailoring Plasmon Coupling in Self-Assembled One-Dimensional Au Nanoparticle Chains through Simultaneous Control of Size and Gap Separation. J. Phys. Chem. Lett. 2013, 4, 2147-2152.

(36) Nooney, R. I.; Stranik, O.; McDonagh, C.; MacCraith, B. D. Optimization of Plasmonic Enhancement of Fluorescence on Plastic Substrates. Langmuir 2008, 24, 11261-11267.

(37) Martinsson, E.; Otte, M. A.; Shahjamali, M. M.; Sepulveda, B.; Aili, D. Substrate Effect on the Refractive Index Sensitivity of Silver Nanoparticles. J. Phys. Chem. C 2014, 118, 24680-24687.

(38) Aili, D.; Stevens, M. M. Bioresponsive peptide-inorganic hybrid nanomaterials. Chem. Soc. Rev. 2010, 39, 3358-3370.

(39) Yguerabide, J.; Yguerabide, E. E. Light-Scattering Submicroscopic Particles as Highly Fluorescent Analogs and Their Use as Tracer Labels in Clinical and Biological Applications. Anal. Biochem. 1998, 262, 137-156.

(40) Lahr, S. J.; Engel, D. E.; Stayrook, S. E.; Maglio, O.; North, B.; Geremia, S.; Lombardi, A.; DeGrado, W. F. Analysis and Design of Turns in $\alpha$-Helical Hairpins. J. Mol. Biol. 2005, 346, 1441-1454.

(41) Dassult Systemes Biovia. Discovery Studio Modeling Environment; Dassault Systemes: San Diego, 2015.

(42) Frisch, M.; Trucks, G.; Schlegel, H. B.; Scuseria, G.; Robb, M.; Cheeseman, J.; Scalmani, G.; Barone, V.; Mennucci, B.; Petersson, G. Gaussian 09, revision D. 01; Gaussian, Inc.: Wallingford CT, 2009.

(43) Breneman, C. M.; Wiberg, K. B. Determining atom-centered monopoles from molecular electrostatic potentials. The need for high sampling density in formamide conformational analysis. J. Comput. Chem. 1990, 11, 361-373.

(44) Schüttelkopf, A. W.; Van Aalten, D. M. F. PRODRG: a tool for high-throughput crystallography of protein-ligand complexes. Acta Crystallogr., Sect. D: Biol. Crystallogr. 2004, 60, 1355-1363.

(45) Van Der Spoel, D.; Lindahl, E.; Hess, B.; Groenhof, G.; Mark, A. E.; Berendsen, H. J. C. GROMACS: fast, flexible, and free. J. Comput. Chem. 2005, 26, 1701-1718.

(46) van Gunsteren, W. F.; Billeter, S. R.; Eising, A. A.; Hünenberger, P. H.; Krüger, P.; Mark, A. E.; Scott, W. R.; Tironi, I. G. Biomolecular Simulation: The \{GROMOS96\} Manual and User Guide; Biomos, 1996.

(47) Bussi, G.; Donadio, D.; Parrinello, M. Canonical sampling through velocity rescaling. J. Chem. Phys. 2007, 126, 014101.

(48) Parrinello, M.; Rahman, A. Polymorphic transitions in single crystals: A new molecular dynamics method. J. Appl. Phys. 1981, 52, $7182-7190$

(49) Hess, B.; Bekker, H.; Berendsen, H. J. C.; Fraaije, J. G. E. M. LINCS: a linear constraint solver for molecular simulations. J. Comput. Chem. 1997, 18, 1463-1472.

(50) Darden, T.; York, D.; Pedersen, L. Particle mesh Ewald: An N $\log (\mathrm{N})$ method for Ewald sums in large systems. J. Chem. Phys. 1993, 98, 10089-10092.

(51) Wallace, A. C.; Laskowski, R. A.; Thornton, J. M. LIGPLOT: a program to generate schematic diagrams of protein-ligand interactions. Protein Eng., Des. Sel. 1995, 8, 127-134. 